\title{
New assistive devices for stroke rehabilitation
}

$\mathrm{R}$ educed mobility in the limbs and extremities represents a major burden for people recovering from stroke, and loss of arm function can be particularly disabling. As demonstrated by two recently published studies, the key to improving rehabilitation outcomes might be found in new assistive technologies, such as robotic exoskeletons and brain-machine interfaces.

In the first study, published in The Lancet Neurology, Verena Klamroth-Marganska and colleagues describe how the ARMin exoskeleton can facilitate the rehabilitation of hemiparesis caused by cerebrovascular accident. "Therapy robots have the potential to enhance recovery of a paralyzed arm or leg beyond what seems to be possible with conventional therapies," she explains.

The ARMin system fits around the affected hand, arm and shoulder to facilitate patients' movements during virtual reality games or activities based on daily living. Klamroth-Marganska's team randomly assigned 77 stroke survivors with moderate or severe hemiparesis to physical therapy assisted by the ARMin system, or to conventional rehabilitation without assistive technologies.

Both groups showed improvement over 8 weeks of therapy, but those using the ARMin exoskeleton recovered more arm mobility than did the conventional rehabilitation group, though the overall size of this effect was modest. In addition, the ARMin system was less effective than conventional therapy at rebuilding arm strength, perhaps because the exoskeleton was too supportive, thus reducing muscle involvement during therapy.

According to Klamroth-Marganska, these results limit the overall clinical value of the ARMin system, but further exploration of this device for the most severely impaired stroke survivors is planned. "This patient group particularly benefited from robotic therapy, which might offer a new mode of therapy for those patients who have the poorest prognosis and the lowest probability of recovery," she says.
For some stroke survivors, therapies based on rehearsal of voluntary movements might never be effective. In a paper published in Neurorehabilitation and Neural Repair, Marc Slutzky and colleagues argue that lost arm function after stroke is not only dependent on lost sensation and strength, but on abnormal coactivation of muscles as well.

Synergy between some muscle groups is important for normal function, such as when the upper arm and forearm muscles work together to reach out and grasp an object. After a stroke, however, muscles that are normally activated independently can become inappropriately entwined, leading to rigid or stereotypical limb movements. As Slutzky points out, "while many approaches to rehabilitation of movement after stroke have been developed, only one has previously targeted abnormal coactivation."

Slutzky and colleagues designed a myoelectric computer interface (MCI) that allowed participants to move a computer cursor by flexing and relaxing their muscles. The MCI measured muscle activity via electrodes placed around the arm and shoulder: independent flexion of muscles moved the cursor in a single direction, and coactivation of different muscles moved the cursor diagonally. The researchers asked patients with abnormal coactivation to move the cursor toward targets in one direction, not along the diagonal. "The patients could only acquire the targets if they learned to decouple their muscles," Slutzky explains.

Slutzky and colleagues trained five healthy participants and five individuals with stroke-induced hemiparesis to use the MCI. All participants were able to learn the task, but the stroke survivors required several training sessions to match the early success of the healthy participants.

After 6 weeks of training on the task, abnormal coactivation had significantly decreased in the participants with stroke, and four of the five stroke survivors reported subjective improvements in arm

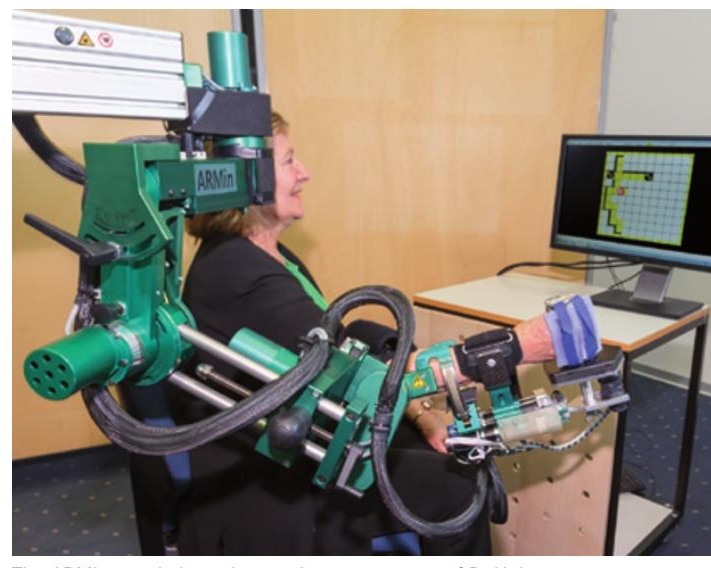

The ARMin exoskeleton in use. Image courtesy of D. Heinz.

function during daily activities. "The MCI directly targets the patients' intrinsic control problem," says Slutzky, and so the therapy's effects might transfer particularly well to a wide range of movements.

Like the ARMin exoskeleton, the MCI requires further development. "Ultimately, we envision that the MCI could be used in parallel with other modes of therapy," says Slutzky. "Because it only requires electromyographic recordings and a computer, it could be designed for widespread use outside research settings, such as in patients' homes."

The full potential of this device will not be clear until it is tested in more patients undergoing more-extensive training. Nevertheless, the MCI and the ARMin systems illustrate the potential for new assistive technologies to improve the lives of millions worldwide.

\section{Alex Chase}

\footnotetext{
Original article Klamroth-Marganska, V. et al. Threedimensional, task-specific robot therapy of the arm after stroke: a multicentre, parallel-group randomised trial. Lancet Neurol. doi:10.1016/S1474-4422(13)70305-3 | Wright, Z. A., Rymer, W. Z. \& Slutzky, M. W. Reducing abnormal muscle coactivation after stroke using a myoelectric-computer interface: a pilot study. Neurorehabil. Neural Repair doi:10.1177/1545968313517751

Further reading Kirchner, E. A., Tabie, M. \& Seeland, A. Multimodal movement prediction-towards an individual assistance of patients. PLoS ONE 9, e85060 (2014)
} 